\title{
ON AMALGAMATED BANACH ALGEBRAS
}

\author{
H. POURMAHMOOD AGHABABA AND N. SHIRMOHAMMADI
}

\begin{abstract}
Let $A$ and $B$ be Banach algebras, $\theta: A \rightarrow B$ be a continuous Banach algebra homomorphism and $I$ be a closed ideal in $B$. Then the direct sum of $A$ and $I$ with respect to $\theta$, denoted $A \bowtie^{\theta} I$, with a special product becomes a Banach algebra which is called the amalgamated Banach algebra. In this paper, among other things, we compute the topological centre of $A \bowtie^{\theta} I$ in terms of that of $A$ and $I$. Using this, we provide a characterization of the Arens regularity of $A \bowtie^{\theta} I$. Then we determine the character space of $A \bowtie^{\theta} I$ in terms of that of $A$ and $I$. Moreover, we study the weak amenability of $A \bowtie^{\theta} I$.
\end{abstract}

\section{INTRODUCTION}

Let $A$ and $B$ be Banach algebras, $\theta: A \rightarrow B$ be a continuous Banach algebra homomorphism, which without loss of generality we can assume that $\|\theta\| \leq 1$, and let $I$ be a closed ideal in $B$. We consider the Banach algebra $A \bowtie^{\theta} I=\{(a, i): a \in A, i \in I\}$, the $l^{1}$-direct sum of $A$ and $I$, with the following product formula:

$$
(a, i) \cdot\left(a^{\prime}, i^{\prime}\right)=\left(a a^{\prime}, \theta(a) i^{\prime}+i \theta\left(a^{\prime}\right)+i i^{\prime}\right)
$$

$A \bowtie^{\theta} I$ is called the amalgamation of $A$ with $B$ along $I$ with respect to $\theta$.

The algebraic version of amalgamated Banach algebras are studied by many algebraists, see for example [7, 8, 9, 10, 20].

A special case of amalgamated Banach algebras, with $I=B$, is studied by some authors, see [1, 3, 14, for example. To our knowledge there are no concrete Banach algebra with this structure. While, as Example 2.1 shows, many classes of concrete Banach algebras can be represented as amalgamated Banach algebras.

The organization of paper is as follows. In Section 2 of this paper, after presenting some examples of amalgamated Banach algebras we establish some primary properties of these algebras. In Sections 3 and 4, we characterize the second dual and topological centres of $A \bowtie^{\theta} I$ as well as its Arens regularity. In Section 5 we characterize the character space of $A \bowtie^{\theta} I$. Finally, Section 6 is devoted to the investigation of the weak amenability of $A \bowtie^{\theta} I$.

The first author is supported by University of Tabriz.

2000 Mathematics Subject Classification. 46H25, 16E40, $13 \mathrm{~B} 02$.

Key words and phrases. Banach modules, Topological centre, Weak amenability. 


\section{Some Examples and Primary Properties}

We commence this section with listing a number of concrete Banach algebras that have the amalgamated structure.

Example 2.1. $\quad$ (i) If $\theta=0$, then $A \bowtie^{0} I$ is nothing but the cartesian product of $A$ and $I$.

(ii) Let $A$ be a non-unital Banach algebra. Then the unitization of $A$, i.e. $A^{\#}=\mathbb{C} \oplus A$, is the amalgamation of $\mathbb{C}$ with $A^{\#}$ along $A$ with respect to the homomorphism $\theta: \mathbb{C} \rightarrow A^{\#}$ defined by $\theta(\lambda)=(\lambda, 0)$.

(iii) Let $A$ be a Banach algebra and $X$ be a Banach $A$-bimodule. Then the module extension Banach algebra $\mathcal{S}=A \oplus X$ is the amalgamation of $A$ with $\mathcal{S}$ along $X$ with respect to the injection $\theta: A \rightarrow \mathcal{S}$ defined by $\theta(a)=(a, 0)$. Notice that the class of module extension Banach algebras include the class of triangular Banach algebras.

(iv) Let $A$ be a Banach algebra and $\phi$ be a nonzero character on $A$. Then $A \bowtie^{\phi} \mathbb{C}$ is the Banach algebra with the underlying Banach space $A \oplus \mathbb{C}$ and with the product

$$
(a, \lambda) \cdot\left(a^{\prime}, \lambda^{\prime}\right)=\left(a a^{\prime}, \phi(a) \lambda^{\prime}+\phi\left(a^{\prime}\right) \lambda+\lambda \lambda^{\prime}\right) .
$$

(v) Let $A$ and $B$ be Banach algebras and let $\phi$ be a nonzero character on $A$. Then $A \bowtie^{\theta} B$, the amalgamation of $A$ with $B^{\#}$ along $B$ with respect to the homomorphism $\theta: A \rightarrow B^{\#}$ defined by $\theta(a)=(\phi(a), 0)$, is the Banach algebra with the underlying Banach space $A \oplus_{1} B$, the $l^{1}$-direct sum of $A$ and $B$, and with the following product formula:

$$
(a, b) \cdot\left(a^{\prime}, b^{\prime}\right)=\left(a a^{\prime}, \phi(a) b^{\prime}+\phi\left(a^{\prime}\right) b+b b^{\prime}\right) .
$$

This is a known Banach algebra denoted by $A \oplus_{\phi} B$, called the $\phi$-Lau product of $A$ and $B$, see [21] for example. This class includes the class of Lau algebras introduced in [17].

(vi) One of the other interesting examples is the semidirect product of Banach algebras. Indeed, let $B$ be a Banach algebra, $A$ be a closed subalgebra of $B$ and $I$ be a closed ideal in $B$. If $\iota: A \rightarrow B$ is the inclusion map, the amalgamated Banach algebra $C=A \bowtie^{\iota} I$ is $A \ltimes I$, the semidirect product of $A$ and $I[6$, Page 8] (as far as we know, the term "semidirect product" in the theory of (commutative) Banach algebras is introduced and studied by Thomas in [22]). We give an important class of Banach algebras which can be recognized as a semidirect product. Let $A$ be a dual Banach algebra with predual $A_{*}$ and consider $A^{* *}$, the second dual of $A$ equipped with either first or second Arens product (see Section 3 for definitions). It is shown in [6, Theorem 2.15] that $A^{* *}=A \ltimes A_{*}^{\perp}$, where $A_{*}^{\perp}=\left\{F \in A^{* *}: F=0\right.$ on $\left.A_{*}\right\}$. We remark that every von Neumann algebra, the measure algebra $M(G)$ of a locally compact group $G$, and the second dual of an Arens regular Banach algebra are examples of dual Banach algebras. Also the measure algebra of a locally compact group $G$ has a natural semidirect product structure. In fact we have $M(G)=l^{1}(G) \ltimes M_{c}(G)$, where $l^{1}(G)$ and $M_{c}(G)$ denote the space of discrete measures and continuous measures in $M(G)$, respectively. 
In the following proposition, we have collected some basic properties of the Banach algebra $A \bowtie^{\theta} I$.

Proposition 2.1. Let $A \bowtie^{\theta} I$ be the amalgamation of $A$ with $B$ along $I$ with respect to $\theta$.

(i) $A \cong A \times\{0\}$ is a closed subalgebra of $A \bowtie^{\theta} I, I \cong\{0\} \times I$ is a closed ideal in $A \bowtie^{\theta} I$ and $\frac{A \bowtie^{\theta} I}{I} \cong A$.

(ii) $A \bowtie^{\theta} I$ is commutative if and only if $A$ and $\theta(A)+I$ are commutative.

(iii) $(a, i)$ is an identity for $A \bowtie^{\theta} I$ if and only if $a=1_{A}, i^{2}=i, i \in \operatorname{Ann}_{I}(\theta(A))$ and $\theta(a)+i=1_{\theta(A)+I}$, where $\operatorname{Ann}_{I}(\theta(A))=\{j \in I: j \theta(a)=\theta(a) j=0$ for all $a \in A\}$.

(iv) $\left(\left(a_{\alpha}, i_{\alpha}\right)\right)_{\alpha}$ is a (bounded) left (right, or two-sided) approximate identity for $A \bowtie^{\theta} I$ if and only if $\left(a_{\alpha}\right)_{\alpha}$ is a (bounded) left (right, or two-sided) approximate identity for $A$, $\left(\theta\left(a_{\alpha}\right)+i_{\alpha}\right)_{\alpha}$ is a (bounded) left (right, or two-sided) approximate identity for $\theta(A)+I$ and $i_{\alpha} \theta(a) \rightarrow 0$ for all $a \in A$.

(v) If $A \bowtie^{\theta} I$ is commutative, then $A \bowtie^{\theta} I$ is regular if and only if both $A$ and $I$ are regular (see [16, Definition 4.2.1]).

(vi) $A \bowtie^{\theta} I$ is amenable if and only if $A$ and $I$ are amenable (see [19, Definition 2.1.9]).

Proof. All of the parts (i)-(iv) can be easily checked. The part (v) follows from Theorems 4.2.6 and 4.3.8 of [16], since $\frac{A \bowtie^{\theta} I}{I} \cong A$. Finally, (vi) follows from Corollary 2.3.2, Theorem 2.3.7 and Theorem 2.3.10 of [19].

Corollary 2.2. Let $A \bowtie^{\mathrm{id}} A$ be the amalgamation of $A$ with $A$ along $A$ with respect to the identity map id on $A$.

(i) $A \bowtie^{\text {id }} A$ is commutative if and only if $A$ is commutative.

(ii) $(a, b)$ is an identity for $A \bowtie^{\text {id }} A$ if and only if $a=1_{A}$ and $b=0$.

(iii) $\left(\left(a_{\alpha}, b_{\alpha}\right)\right)_{\alpha}$ is a (bounded) left (right, or two-sided) approximate identity for $A \bowtie^{\text {id }} A$ if and only if $\left(a_{\alpha}\right)_{\alpha}$ is a (bounded) left (right, or two-sided) approximate identity for $A$ and $b_{\alpha} \rightarrow 0$.

(v) If $A$ is commutative, then $A \bowtie^{\mathrm{id}} A$ is regular if and only if $A$ is regular.

(vi) $A \bowtie^{\text {id }} A$ is amenable if and only if $A$ is amenable.

\section{The First and Second Arens Products on $\left(A \bowtie^{\theta} I\right)^{* *}$}

Let $A$ be a Banach algebra and $A^{*}$ and $A^{* *}$ be the first and second duals of $A$, respectively. Let $a \in A, f \in A^{*}$. Then $a \cdot f$ and $f \cdot a \in A^{*}$ are defined by

$$
\langle a \cdot f, b\rangle=\langle f, b a\rangle, \quad\langle f \cdot a, b\rangle=\langle f, a b\rangle \quad(b \in A),
$$

making $A^{*}$ an $A$-bimodule. Similarly, $A^{* *}$ is an $A$-bimodule.

There are two natural products on $A^{* *}$, called the first and second Arens products, and are denoted by $\square$ and $\diamond$, respectively. They were introduced by Arens [2] (for more details the 
reader is refereed to [4]). We recall briefly the definitions. For $f \in A^{*}$ and $F \in A^{* *}$ define $f \cdot F \in A^{*}$ and $F \cdot f \in A^{*}$ by

$$
\langle f \cdot F, a\rangle=\langle F, a \cdot f\rangle, \quad\langle F \cdot f, a\rangle=\langle F, f \cdot a\rangle \quad(a \in A) .
$$

Now, for $F, G \in A^{* *}$, define $F \square G \in A^{* *}$ and $F \diamond G \in A^{* *}$ by

$$
\langle F \square G, f\rangle=\langle F, G \cdot f\rangle, \quad\langle F \diamond G, f\rangle=\langle G, f \cdot F\rangle \quad\left(f \in A^{*}\right) .
$$

Then $\left(A^{* *}, \square\right)$ and $\left(A^{* *}, \diamond\right)$ are Banach algebras containing $A$ as a closed subalgebra.

Proposition 3.1. $\left(A \bowtie^{\theta} I\right)^{*}$ is isometrically isomorphic to $A^{*} \oplus_{\infty} I^{*}$ as Banach spaces. The isomorphism $\Psi: A^{*} \oplus_{\infty} I^{*} \rightarrow\left(A \bowtie^{\theta} I\right)^{*}$ is given by

$$
\langle(a, i), \Psi(f, g)\rangle=f(a)+g(i) \quad\left((a, i) \in A \bowtie^{\theta} I,(f, g) \in A^{*} \oplus_{\infty} I^{*}\right) .
$$

Proof. The proof is straightforward and is omitted.

Corollary 3.2. $\left(A \bowtie^{\theta} I\right)^{* *}$ is isometrically isomorphic to $A^{* *} \oplus_{1} I^{* *}$ as Banach spaces.

Now we explore the left and right module actions of $A \bowtie^{\theta} I$ on $\left(A \bowtie^{\theta} I\right)^{*}$ in order to provide a characterization of the first and second Arens product on $\left(A \bowtie^{\theta} I\right)^{* *}$.

Theorem 3.3. Let $A \bowtie^{\theta} I$ be the amalgamation of $A$ with $B$ along $I$ with respect to $\theta$. Then

$$
\left(\left(A \bowtie^{\theta} I\right)^{* *}, \square\right)=\left(A^{* *}, \square\right) \bowtie^{\theta^{* *}}\left(I^{* *}, \square\right)
$$

where $\theta^{* *}$ is the second adjoint of $\theta$.

Proof. Let $a, b \in A, i, j \in I, f \in A^{*}$ and $g \in I^{*}$. Then $(f, g) \cdot(a, i) \in\left(A \bowtie^{\theta} I\right)^{*}$ can be calculated as follows:

$$
\begin{aligned}
\langle(b, j),(f, g) \cdot(a, i)\rangle & =\langle(a, i) \cdot(b, j),(f, g)\rangle \\
& =\langle(a b, i \theta(b)+\theta(a) j+i j),(f, g)\rangle \\
& =\langle a b, f\rangle+\langle\theta(a) j, g\rangle+\langle i \theta(b), g\rangle+\langle i j, g\rangle \\
& =\langle b, f \cdot a\rangle+\langle j, g \cdot \theta(a)\rangle+\langle\theta(b), g \cdot i\rangle+\langle j, g \cdot i\rangle \\
& =\langle b, f \cdot a\rangle+\langle j, g \cdot \theta(a)\rangle+\left\langle b, \theta^{*}(g \cdot i)\right\rangle+\langle j, g \cdot i\rangle \\
& =\left\langle b, f \cdot a+\theta^{*}(g \cdot i)\right\rangle+\langle j, g \cdot(\theta(a)+i)\rangle,
\end{aligned}
$$

and so

$$
(f, g) \cdot(a, i)=\left(f \cdot a+\theta^{*}(g \cdot i), g \cdot(\theta(a)+i)\right)
$$


Further, let $F_{1} \in A^{* *}, F_{2} \in I^{* *}$. Then, in order to calculate $\left(F_{1}, F_{2}\right) \cdot(f, g) \in\left(A \bowtie^{\theta} I\right)^{*}$, one has

$$
\begin{aligned}
\left\langle(a, i),\left(F_{1}, F_{2}\right) \cdot(f, g)\right\rangle & =\left\langle(f, g) \cdot(a, i),\left(F_{1}, F_{2}\right)\right\rangle \\
& =\left\langle\left(f \cdot a+\theta^{*}(g \cdot i), g \cdot(\theta(a)+i)\right),\left(F_{1}, F_{2}\right)\right\rangle \\
& =\left\langle f \cdot a+\theta^{*}(g \cdot i), F_{1}\right\rangle+\left\langle g \cdot(\theta(a)+i), F_{2}\right\rangle \\
& =\left\langle a, F_{1} \cdot f\right\rangle+\left\langle i, \theta^{* *}\left(F_{1}\right) \cdot g\right\rangle+\left\langle\theta(a), F_{2} \cdot g\right\rangle+\left\langle i, F_{2} \cdot g\right\rangle \\
& =\left\langle a, F_{1} \cdot f+\theta^{*}\left(F_{2} \cdot g\right)\right\rangle+\left\langle i, F_{2} \cdot g+\theta^{* *}\left(F_{1}\right) \cdot g\right\rangle \\
& =\left\langle(a, i),\left(F_{1} \cdot f+\theta^{*}\left(F_{2} \cdot g\right), F_{2} \cdot g+\theta^{* *}\left(F_{1}\right) \cdot g\right)\right\rangle .
\end{aligned}
$$

Thus

$$
\left(F_{1}, F_{2}\right) \cdot(f, g)=\left(F_{1} \cdot f+\theta^{*}\left(F_{2} \cdot g\right), F_{2} \cdot g+\theta^{* *}\left(F_{1}\right) \cdot g\right) .
$$

Now for $\left(F_{1}, F_{2}\right),\left(G_{1}, G_{2}\right) \in\left(A \bowtie^{\theta} I\right)^{* *} \cong A^{* *} \oplus_{1} I^{* *}$ and $(f, g) \in\left(A \bowtie^{\theta} I\right)^{*} \cong A^{*} \oplus_{\infty} I^{*}$, using (3.1) and (3.2), we have

$$
\begin{aligned}
\left\langle(f, g),\left(F_{1}, F_{2}\right) \square\left(G_{1}, G_{2}\right)\right\rangle & =\left\langle\left(G_{1}, G_{2}\right) \cdot(f, g),\left(F_{1}, F_{2}\right)\right\rangle \\
& =\left\langle\left(G_{1} \cdot f+\theta^{*}\left(G_{2} \cdot g\right), G_{2} \cdot g+\theta^{* *}\left(G_{1}\right) \cdot g\right),\left(F_{1}, F_{2}\right)\right\rangle \\
& =\left\langle G_{1} \cdot f+\theta^{*}\left(G_{2} \cdot g\right), F_{1}\right\rangle+\left\langle G_{2} \cdot g+\theta^{* *}\left(G_{1}\right) \cdot g, F_{2}\right\rangle \\
& =\left\langle f, F_{1} \square G_{1}\right\rangle+\left\langle G_{2} \cdot g, \theta^{* *}\left(F_{1}\right)\right\rangle+\left\langle g, F_{2} \square G_{2}\right\rangle+\left\langle\theta^{* *}\left(G_{1}\right) \cdot g, F_{2}\right\rangle \\
& =\left\langle f, F_{1} \square G_{1}\right\rangle+\left\langle g, \theta^{* *}\left(F_{1}\right) \square G_{2}\right\rangle+\left\langle g, F_{2} \square G_{2}\right\rangle+\left\langle g, F_{2} \square \theta^{* *}\left(G_{1}\right)\right\rangle \\
& =\left\langle f, F_{1} \square G_{1}\right\rangle+\left\langle g, \theta^{* *}\left(F_{1}\right) \square G_{2}+F_{2} \square G_{2}+F_{2} \square \theta^{* *}\left(G_{1}\right)\right\rangle \\
& =\left\langle(f, g),\left(F_{1} \square G_{1}, \theta^{* *}\left(F_{1} \square G_{1}\right)+\theta^{* *}\left(F_{1}\right) \square G_{2}+F_{2} \square G_{2}+F_{2} \square \theta^{* *}\left(G_{1}\right)\right\rangle .\right.
\end{aligned}
$$

Therefore,

$$
\left(F_{1}, F_{2}\right) \square\left(G_{1}, G_{2}\right)=\left(F_{1} \square G_{1}, \theta^{* *}\left(F_{1}\right) \square G_{2}+F_{2} \square G_{2}+F_{2} \square \theta^{* *}\left(G_{1}\right)\right) .
$$

This completes the proof.

Similarly, as notation in the proof of Theorem 3.3, one can show that

$$
\begin{gathered}
(a, i) \cdot(f, g)=\left(a \cdot f+\theta^{*}(i \cdot g),(\theta(a)+i) \cdot g\right), \\
(f, g) \cdot\left(F_{1}, F_{2}\right)=\left(f \cdot F_{1}+\theta^{*}\left(g \cdot F_{2}\right), g \cdot F_{2}+g \cdot \theta^{* *}\left(F_{1}\right)\right), \\
\left(F_{1}, F_{2}\right) \diamond\left(G_{1}, G_{2}\right)=\left(F_{1} \diamond G_{1}, \theta^{* *}\left(F_{1}\right) \diamond G_{2}+F_{2} \diamond \theta^{* *}\left(G_{1}\right)+F_{2} \diamond G_{2}\right) .
\end{gathered}
$$

Therefore,

$$
\left(\left(A \bowtie^{\theta} I\right)^{* *}, \diamond\right)=\left(A^{* *}, \diamond\right) \bowtie^{\theta^{* *}}\left(I^{* *}, \diamond\right) .
$$




\section{Topological Centres}

Let $A$ be a Banach algebra and $X$ a Banach $A$-bimodule. Then $X^{* *}$ is canonically an $\left(A^{* *}, \square\right)$ bimodule $\left(\left(A^{* *}, \diamond\right)\right.$-bimodule), see 4 , Page 248$]$. Let $x^{\prime \prime} \in X^{* *}$ and let $L_{x^{\prime \prime}}, R_{x^{\prime \prime}}:\left(A^{* *}, \square\right) \rightarrow X^{* *}$ be the left and right multiplication operators, respectively, i.e.

$$
L_{x^{\prime \prime}}\left(a^{\prime \prime}\right)=x^{\prime \prime} \square a^{\prime \prime}=\lim _{\beta} \lim _{\alpha} x_{\beta} a_{\alpha} \quad \text { and } \quad R_{x^{\prime \prime}}\left(a^{\prime \prime}\right)=a^{\prime \prime} \square x^{\prime \prime}=\lim _{\alpha} \lim _{\beta} a_{\alpha} x_{\beta} \quad\left(a^{\prime \prime} \in A^{* *}\right),
$$

where $\left(a_{\alpha}\right)$ and $\left(x_{\beta}\right)$ are nets in $A^{* *}$ and $X^{* *}$, respectively, in such a way that $a_{\alpha} \rightarrow a^{\prime \prime}$ in the $w^{*}$-topology of $A^{* *}$ and $x_{\beta} \rightarrow x^{\prime \prime}$ in the $w^{*}$-topology of $X^{* *}$.

Likewise let $\mathcal{L}_{x^{\prime \prime}}, \mathcal{R}_{x^{\prime \prime}}:\left(A^{* *}, \diamond\right) \rightarrow X^{* *}$ be the left and right multiplication operators, respectively, i.e.

$$
\mathcal{L}_{x^{\prime \prime}}\left(a^{\prime \prime}\right)=x^{\prime \prime} \diamond a^{\prime \prime}=\lim _{\alpha} \lim _{\beta} x_{\beta} a_{\alpha} \quad \text { and } \quad \mathcal{R}_{x^{\prime \prime}}\left(a^{\prime \prime}\right)=a^{\prime \prime} \diamond x^{\prime \prime}=\lim _{\beta} \lim _{\alpha} a_{\alpha} x_{\beta} \quad\left(a^{\prime \prime} \in A^{* *}\right) .
$$

The left and right topological centres, $Z_{A}^{\ell, t}\left(X^{* *}\right)$ and $Z_{A}^{r, t}\left(X^{* *}\right)$ of $X^{* *}$ are

$$
Z_{A}^{\ell, t}\left(X^{* *}\right)=\left\{x^{\prime \prime} \in X^{* *}: L_{x^{\prime \prime}}=\mathcal{L}_{x^{\prime \prime}}\right\}=\left\{x^{\prime \prime} \in X^{* *}: x^{\prime \prime} \square a^{\prime \prime}=x^{\prime \prime} \diamond a^{\prime \prime}, \forall a^{\prime \prime} \in A^{* *}\right\},
$$

and

$$
Z_{A}^{r, t}\left(X^{* *}\right)=\left\{x^{\prime \prime} \in X^{* *}: R_{x^{\prime \prime}}=\mathcal{R}_{x^{\prime \prime}}\right\}=\left\{x^{\prime \prime} \in X^{* *}: a^{\prime \prime} \square x^{\prime \prime}=a^{\prime \prime} \diamond x^{\prime \prime}, \forall a^{\prime \prime} \in A^{* *}\right\},
$$

respectively. Then we say that $X$ is Arens regular (as an $A$-bimodule) or $A$ acts regularly on $X$ if

$$
Z_{A}^{\ell, t}\left(X^{* *}\right)=Z_{A}^{r, t}\left(X^{* *}\right)=X^{* *}
$$

and $X$ is left strongly Arens irregular if $Z_{A}^{\ell, t}\left(X^{* *}\right)=X$, right strongly Arens irregular if $Z_{A}^{r, t}\left(X^{* *}\right)=X$, and strongly Arens irregular if it is both left and right strongly Arens irregular.

If $X=A$, we will use the common notation $Z_{t}^{\ell}\left(A^{* *}\right)$ and $Z_{t}^{r}\left(A^{* *}\right)$ in place of $Z_{A}^{\ell, t}\left(A^{* *}\right)$ and $Z_{A}^{r, t}\left(A^{* *}\right)$, respectively.

Now, let $B$ be a Banach algebra, $I$ be a closed ideal in $B$ and $\theta: A \rightarrow B$ be a continuous Banach algebra homomorphism. Then we define

$$
Z_{\theta^{* *}}^{\ell}\left(A^{* *}\right)=\left\{F \in Z_{t}^{\ell}\left(A^{* *}\right): \theta^{* *}(F) \in Z_{I}^{\ell}\left(\theta(A)^{* *}\right)\right\}
$$

note that $\theta(A)^{* *}=\theta^{* *}\left(A^{* *}\right)([4$, Page 251]), where

$$
Z_{I}^{\ell}\left(\theta(A)^{* *}\right)=\left\{F \in \theta(A)^{* *}: L_{F}=\mathcal{L}_{F} \text { on } I^{* *}\right\}=\left\{F \in \theta(A)^{* *}: F \square G=F \diamond G, \forall G \in I^{* *}\right\} .
$$

Theorem 4.1. With above notation and assumptions, one has

$$
Z_{t}^{\ell}\left(\left(A \bowtie^{\theta} I\right)^{* *}\right)=Z_{t}^{\ell}\left(A^{* *} \bowtie^{\theta^{* *}} I^{* *}\right)=Z_{\theta^{* *}}^{\ell}\left(A^{* *}\right) \bowtie^{\theta^{* *}}\left(Z_{t}^{\ell}\left(I^{* *}\right) \cap Z_{\theta(A)}^{\ell, t}\left(I^{* *}\right)\right) .
$$


Proof. Let $\left(F_{1}, G_{1}\right) \in Z_{t}^{\ell}\left(A^{* *} \bowtie^{\theta^{* *}} I^{* *}\right)$. Then $L_{\left(F_{1}, G_{1}\right)}=\mathcal{L}_{\left(F_{1}, G_{1}\right)}$ if and only if for all $\left(F_{2}, G_{2}\right) \in$ $A^{* *} \bowtie^{\theta^{* *}} I^{* *}$,

$$
\left(F_{1}, G_{1}\right) \square\left(F_{2}, G_{2}\right)=\left(F_{1}, G_{1}\right) \diamond\left(F_{2}, G_{2}\right)
$$

if and only if

$\left(F_{1} \square F_{2}, \theta^{* *}\left(F_{1}\right) \square G_{2}+G_{1} \square \theta^{* *}\left(F_{2}\right)+G_{1} \square G_{2}\right)=\left(F_{1} \diamond F_{2}, \theta^{* *}\left(F_{1}\right) \diamond G_{2}+G_{1} \diamond \theta^{* *}\left(F_{2}\right)+G_{1} \diamond G_{2}\right)$, if and only if $L_{F_{1}}=\mathcal{L}_{F_{1}}, L_{\theta^{* *}\left(F_{1}\right)}=\mathcal{L}_{\theta^{* *}\left(F_{1}\right)}$ on $I^{* *}, L_{G_{1}}=\mathcal{L}_{G_{1}}$ on $I^{* *}$ and $\theta(A)^{* *}$. Hence,

$$
\left(F_{1}, G_{1}\right) \in Z_{\theta^{* *}}^{\ell}\left(A^{* *}\right) \bowtie^{\theta^{* *}}\left(Z_{t}^{\ell}\left(I^{* *}\right) \cap Z_{\theta(A)}^{\ell, t}\left(I^{* *}\right)\right) .
$$

Using above theorem we characterize Arens regularity and strong Arens irregularity of $A \bowtie^{\theta} I$.

Corollary 4.2. $A \bowtie^{\theta} I$ is Arens regular if and only if $A$ and $I$ are Arens regular, $\theta(A)$ acts regularly on $I$ and $I$ acts regularly on $\theta(A)$.

Corollary 4.3. $A \bowtie^{\theta} I$ is strongly Arens irregular if and only if $A$ and $I$ are strongly Arens irregular, $I$ acts strongly irregular on $\theta(A)$ and $\theta(A)$ acts strongly irregular on $I$.

In the following example we determine topological centres of some amalgamated Banach algebras.

Example 4.1. Keep the notation of Example 2.1.

(i) If $\theta=0$, then

$$
Z_{t}^{\ell}\left((A \oplus I)^{* *}\right)=Z_{t}^{\ell}\left(\left(A \bowtie^{0} I\right)^{* *}\right)=Z_{t}^{\ell}\left(A^{* *}\right) \bowtie^{0} Z_{t}^{\ell}\left(I^{* *}\right)=Z_{t}^{\ell}\left(A^{* *}\right) \oplus Z_{t}^{\ell}\left(I^{* *}\right) .
$$

(ii) $Z_{t}^{\ell}\left(\left(A^{\#}\right)^{* *}\right)=\mathbb{C} \oplus Z_{t}^{\ell}\left(A^{* *}\right)=Z_{t}^{\ell}\left(A^{* *}\right)^{\#}$.

(iii) ([11]) Let $\mathcal{S}=A \oplus X$ be the module extension Banach algebra corresponding $A$ and $X$. Then, by noting that $\theta$ is the canonical embedding of $A$ into $\mathcal{S}$, and $I=X$ with $X^{2}=0$, we have

$$
Z_{\theta^{* *}}^{\ell}\left(A^{* *}\right)=\left\{F \in Z_{t}^{\ell}\left(A^{* *}\right): F \in Z_{X}^{\ell}\left(A^{* *}\right)\right\}=Z_{t}^{\ell}\left(A^{* *}\right) \cap Z_{X}^{\ell}\left(A^{* *}\right),
$$

and

$$
Z_{t}^{\ell}\left(I^{* *}\right) \cap Z_{\theta(A)}^{\ell, t}\left(I^{* *}\right)=Z_{t}^{\ell}\left(X^{* *}\right) \cap Z_{A}^{\ell, t}\left(X^{* *}\right)=Z_{t}^{\ell}\left(X^{* *}\right)=X^{* *} .
$$

Therefore,

$$
Z_{t}^{\ell}\left(\mathcal{S}^{* *}\right)=\left(Z_{t}^{\ell}\left(A^{* *}\right) \cap Z_{X}^{\ell}\left(A^{* *}\right)\right) \bowtie^{\theta^{* *}} X^{* *},
$$

that is, $Z_{t}^{\ell}\left(\mathcal{S}^{* *}\right)$ is the module extension Banach algebra corresponding $Z_{t}^{\ell}\left(A^{* *}\right) \cap Z_{X}^{\ell}\left(A^{* *}\right)$ and $X^{* *}$.

(iv) $Z_{t}^{\ell}\left(\left(A \bowtie^{\phi} \mathbb{C}\right)^{* *}\right)=Z_{t}^{\ell}\left(A^{* *}\right) \bowtie^{\phi} \mathbb{C}$. Details are similar to details of the next general case. 
(v) ([21, Corollary 2.13]) For computing $Z_{t}^{\ell}\left(\left(A \oplus_{\phi} B\right)^{* *}\right)$ we note that since $\theta: A \rightarrow B^{\#}$ is defined by $\theta(a)=(\phi(a), 0)=\phi(a)$, one can easily check that $\theta^{* *}: A^{* *} \rightarrow\left(B^{\#}\right)^{* *}=$ $\mathbb{C} \oplus B^{* *}$ is given by $\theta^{* *}(F)=(F(\phi), 0)=F(\phi)=\phi(F)$. So

$$
Z_{\theta^{* *}}^{\ell}\left(A^{* *}\right)=Z_{\phi}^{\ell}\left(A^{* *}\right)=Z_{t}^{\ell}\left(A^{* *}\right)
$$

and

$$
Z_{\theta(A)}^{\ell, t}\left(I^{* *}\right)=Z_{\mathbb{C}}^{\ell, t}\left(B^{* *}\right)=B^{* *}
$$

Whence

$$
Z_{t}^{\ell}\left(\left(A \oplus_{\phi} B\right)^{* *}\right)=Z_{t}^{\ell}\left(A^{* *}\right) \bowtie^{\theta^{* *}} Z_{t}^{\ell}\left(B^{* *}\right)=Z_{t}^{\ell}\left(A^{* *}\right) \oplus_{\phi} Z_{t}^{\ell}\left(B^{* *}\right) .
$$

(vi) If $I=B$ and $\theta$ is surjective, then

$$
Z_{t}^{\ell}\left(\left(A \bowtie^{\theta} B\right)^{* *}\right)=\left(Z_{t}^{\ell}\left(A^{* *}\right) \cap\left(\theta^{* *}\right)^{-1}\left(Z_{t}^{\ell}\left(B^{* *}\right)\right)\right) \bowtie^{\mathrm{id}} Z_{t}^{\ell}\left(B^{* *}\right) .
$$

(vii) Assume that $B=A$. Then

$$
Z_{t}^{\ell}\left(\left(A \bowtie^{\mathrm{id}} I\right)^{* *}\right)=\left(Z_{t}^{\ell}\left(A^{* *}\right) \cap Z_{I}^{\ell}\left(A^{* *}\right)\right) \bowtie^{\mathrm{id}}\left(Z_{t}^{\ell}\left(I^{* *}\right) \cap Z_{A}^{\ell, t}\left(I^{* *}\right)\right) .
$$

(ix) $Z_{t}^{\ell}\left(\left(A \bowtie^{\text {id }} A\right)^{* *}\right)=Z_{t}^{\ell}\left(A^{* *}\right) \bowtie^{\text {id }} Z_{t}^{\ell}\left(A^{* *}\right)$.

(x) Let $B=I=A^{* *}$ and let $\iota: A \rightarrow A^{* *}$ be the cononical injection. Then

$$
Z_{A^{* *}}^{\ell}\left(\iota(A)^{* *}\right)=Z_{A^{* *}}^{\ell}\left(A^{* *}\right)=A^{* *},
$$

and so $Z_{\iota^{* *}}^{\ell}\left(A^{* *}\right)=Z_{t}^{\ell}\left(A^{* *}\right)$. Also $Z_{\iota(A)}^{\ell, t}\left(\left(A^{* *}\right)^{* *}\right)=Z_{A}^{\ell, t}\left(A^{* * * *}\right)=A^{* * * *}$, and thus

$$
Z_{t}^{\ell}\left(\left(A \bowtie^{\iota} A^{* *}\right)^{* *}\right)=Z_{t}^{\ell}\left(A^{* *}\right) \bowtie^{\iota^{* *}} Z_{t}^{\ell}\left(A^{* * * *}\right) .
$$

Example 4.2. By Example 4.1 in mind we have the followings:

(i) The Banach algebra $A \bowtie^{0} I$ is Arens regular if and only if $A$ and $I$ are Arens regular.

(ii) The unitization of $A, A^{\#}$, is Arens regular if and only if $A$ is Arens regular.

(iii) The module extension Banach algebra $\mathcal{S}=A \oplus X$ is Arens regular if and only if $A$ is Arens regular and $A$ acts regularly on $X$.

(iv) $A \bowtie^{\phi} \mathbb{C}$ is Arens regular if and only if $A$ is Arens regular.

(v) $A \oplus_{\phi} B$ is Arens regular if and only if $A$ and $B$ are Arens regular.

(vi) If $I=B$ and $\theta$ is surjective, then $A \bowtie^{\theta} B$ is Arens regular if and only if $A$ and $B$ are Arens regular.

(vii) If $B=A$, then $A \bowtie^{\theta} I$ is Arens regular if and only if $A$ is Arens regular and $A$ and $I$ act regularly on each other.

(ix) The Banach algebra $A \bowtie^{\text {id }} A$ is Arens regular if and only if $A$ is Arens regular.

(x) The Banach algebra $A \bowtie^{\iota} A^{* *}$ is Arens regular if and only if $A$ and $A^{* *}$ are Arens regular. 
Example 4.3. Let $G$ be an infinite locally compact group. Then by [18, Theorem 1] we have $Z_{t}^{\ell}\left(L^{1}(G)^{* *}\right)=L^{1}(G)$, and so

$$
Z_{t}^{\ell}\left(\left(L^{1}(G) \bowtie^{\text {id }} L^{1}(G)\right)^{* *}\right)=Z_{t}^{\ell}\left(L^{1}(G)^{* *}\right) \bowtie^{\text {id }} Z_{t}^{\ell}\left(L^{1}(G)^{* *}\right)=L^{1}(G) \bowtie^{\text {id }} L^{1}(G) .
$$

Therefore, $L^{1}(G) \bowtie^{\text {id }} L^{1}(G)$ is strongly Arens irregular.

\section{Characters of $A \bowtie^{\theta} I$}

In this section, first, we provide a characterization of character space of $A \bowtie^{\theta} I$, and then we calculate the Jacobson radical of $A \bowtie^{\theta} I$ when it is commutative.

Theorem 5.1. Let $\sigma(A) \neq \emptyset$ and $\overline{\theta(A) I \cup I \theta(A)}=I$. Then $\sigma\left(A \bowtie^{\theta} I\right)=E \cup F$, where

$$
E=\{((i \cdot \psi) \circ \theta, \psi): \psi \in \sigma(I), i \in I, \psi(i)=1\}, \quad F=\{(\phi, 0): \phi \in \sigma(A)\} .
$$

Moreover, $E$ is open and $F$ is closed in $\sigma\left(A \bowtie^{\theta} I\right)$.

Proof. Let $(\phi, \psi) \in \sigma\left(A \bowtie^{\theta} I\right)$ and $(a, i),\left(a^{\prime}, i^{\prime}\right) \in A \bowtie^{\theta} I$. Then

$$
\begin{aligned}
\phi\left(a a^{\prime}\right)+\psi\left(\theta(a) i^{\prime}+i \theta(a)+i i^{\prime}\right) & =\left\langle(\phi, \psi),\left(a a^{\prime}, \theta(a) i^{\prime}+i \theta(a)+i i^{\prime}\right)\right\rangle \\
& =\left\langle(\phi, \psi),\left((a, i) \cdot\left(a^{\prime}, i^{\prime}\right)\right\rangle\right. \\
& =(\phi(a)+\psi(i))\left(\phi\left(a^{\prime}\right)+\psi\left(i^{\prime}\right)\right) \\
& =\phi(a) \phi\left(a^{\prime}\right)+\phi(a) \psi\left(i^{\prime}\right)+\psi(i) \phi\left(a^{\prime}\right)+\psi(i) \psi\left(i^{\prime}\right) .
\end{aligned}
$$

By taking $a=a^{\prime}=0$ we see that $\psi \in \sigma(I) \cup\{0\}$. Next by taking $i=i^{\prime}=0$ it follows that $\phi \in \sigma(A) \cup\{0\}$. But from (5.1),$\phi=0$ implies $\psi\left(\theta(a) i^{\prime}\right)+\psi\left(i \theta\left(a^{\prime}\right)\right)=0$ for all $a, a^{\prime} \in A$ and $i, i^{\prime} \in I$, from which it follows that $\psi=0$ on $\theta(A) I \cup I \theta(A)$, and hence $\psi=0$. But this is a contradiction since $(\phi, \psi) \in \sigma\left(A \bowtie^{\theta} I\right)$ and so $(\phi, \psi) \neq(0,0)$. Now we have two cases:

Case I: If $\psi=0$, then $(\phi, \psi)=(\phi, 0)$.

Case II: If $\psi \neq 0$, then by (5.1) we have

$$
\psi\left(\theta(a) i^{\prime}\right)+\psi\left(i \theta\left(a^{\prime}\right)\right)-\psi(i) \phi\left(a^{\prime}\right)-\phi(a) \psi\left(i^{\prime}\right)=0,
$$

which implies (take $a^{\prime}=0, i=0$ ),

$$
\psi\left(\theta(a) i^{\prime}\right)=\phi(a) \psi\left(i^{\prime}\right) \quad\left(a \in A, i^{\prime} \in I\right) .
$$

Choose $i^{\prime} \in I$ such that $\psi\left(i^{\prime}\right)=1$, then $\phi(a)=\psi\left(\theta(a) i^{\prime}\right)=\left(i^{\prime} \cdot \psi\right) \circ \theta(a)$ for all $a \in A$. Therefore, $(\phi, \psi)=\left(\left(i^{\prime} \cdot \psi\right) \circ \theta, \psi\right)$ with $\psi\left(i^{\prime}\right)=1$.

Since the reverse inclusion is easy to check, so we omit its proof.

Now we show that $E$ is open in the $w^{*}$-topology of $\sigma\left(A \bowtie^{\theta} I\right)$ induced from $w^{*}$-topology of $A^{*} \times I^{*}$. Let $\left(\left(i \cdot \psi_{0}\right) \circ \theta, \psi_{0}\right) \in \sigma\left(A \bowtie^{\theta} I\right)$. Then there is $i_{0} \in I$ in such a way that $\psi\left(i_{0}\right) \neq 0$. Let $\varepsilon=\left|\psi\left(i_{0}\right)\right|$. Then

$$
\begin{aligned}
U & =\left\{(\phi, \psi) \in \sigma\left(A \bowtie^{\theta} I\right):\left|(\phi, \psi)\left(0, i_{0}\right)-\left(\left(i \cdot \psi_{0}\right) \circ \theta, \psi_{0}\right)\left(0, i_{0}\right)\right|<\varepsilon\right\} \\
& =\left\{(\phi, \psi) \in \sigma\left(A \bowtie^{\theta} I\right):\left|\psi\left(i_{0}\right)-\psi_{0}\left(i_{0}\right)\right|<\varepsilon\right\},
\end{aligned}
$$


is a neighborhood of $\left(\left(i \cdot \psi_{0}\right) \circ \theta, \psi_{0}\right)$ in the $w^{*}$-topology of $\sigma\left(A \bowtie^{\theta} I\right)$. Since $(\phi, 0) \in U$ leads to the contradiction $\left|\psi_{0}\left(i_{0}\right)\right|<\varepsilon$, it follows that $U \subseteq E$. Therefore, $E$ is open and $F$ is closed.

Corollary 5.2. ([21, Proposition 2.4]) Let $\sigma(A) \neq \emptyset$ and $\phi \in \sigma(A)$. Then

$$
\sigma\left(A \oplus_{\phi} B\right)=\{(\varphi, 0): \varphi \in \sigma(A)\} \cup\{(\phi, \psi): \psi \in \sigma(B)\}=(\sigma(A) \times\{0\}) \cup(\{\phi\} \times \sigma(B)) .
$$

Proof. It is enough to note that $(i \cdot \psi) \circ \theta=\theta$ if $\psi(i)=1$.

Let $A$ be a commutative Banach algebra. The radical of $A, \operatorname{rad} A$, is the intersection of the kernels of all characters of $A$. Also $A$ is called semisimple if $\operatorname{rad} A=\{0\}$.

Theorem 5.3. Let $A \bowtie^{\theta} I$ be commutative, $\sigma(A) \neq \emptyset$ and $\overline{\theta(A) I}=I$. Then $\operatorname{rad}\left(A \bowtie^{\theta} I\right)=$ $\operatorname{rad} A \oplus \operatorname{rad} I$.

Proof. Let $(a, i) \in \operatorname{rad}\left(A \bowtie^{\theta} I\right)$. Then for each $\phi \in \sigma(A), \phi(a)=(\phi, 0)(a, i)=0$, that is, $a \in \operatorname{rad} A$. Now let $\psi \in \sigma(I)$ and $\psi(j)=1$ for some $j \in I$. Then $(j \cdot \psi) \circ \theta$ belongs to $\sigma(A)$ and so $(j \cdot \psi) \circ \theta(a)=0$. Hence

$$
\psi(i)=((j \cdot \psi) \circ \theta, \psi)(a, i)-(j \cdot \psi) \circ \theta(a)=0,
$$

and thus $i \in \operatorname{rad} I$.

Conversely let $a \in \operatorname{rad} A$ and $i \in \operatorname{rad} I$. Then for each $\phi \in \sigma(A),(\phi, 0)(a, i)=\phi(a)=0$ and for each $\psi \in \sigma(I)$,

$$
((j \cdot \psi) \circ \theta, \psi)(a, i)=(j \cdot \psi) \circ \theta(a)+\psi(i)=0 .
$$

Therefore, by Theorem [5.1, $(a, i) \in \operatorname{rad}\left(A \bowtie^{\theta} I\right)$.

Corollary 5.4. Let $A \bowtie^{\theta} I$ be commutative, $\sigma(A) \neq \emptyset$ and $\overline{\theta(A) I}=I$. Then $A \bowtie^{\theta} I$ is semisimple if and only if both $A$ and $I$ are semisimple.

\section{WEAK AmEnABiLity}

Let $A$ be a Banach algebra and $X$ a Banach $A$-bimodule. A derivation from $A$ into $X$ is a bounded linear map satisfying $D(a b)=a \cdot D(b)+D(a) \cdot b$ for all $a, b \in A$. For each $x \in X$ we denote by $\operatorname{ad}_{x}$ the derivation $D(a)=a \cdot x-x \cdot a$ for all $a \in A$, called an inner derivation. We denote by $\mathcal{Z}^{1}(A, X)$ the space of all derivations from $A$ into $X$ and by $\mathcal{B}^{1}(A, X)$ the space of all inner derivations from $A$ into $X$. The first cohomology group of $A$ with coefficients in $X$ is $\mathcal{H}^{1}(A, X)=\mathcal{Z}^{1}(A, X) / \mathcal{B}^{1}(A, X)$. A Banach algebra $A$ is called weakly amenable if $\mathcal{H}^{1}\left(A, A^{*}\right)=0$.

In [12], B. E. Forrest and L. W. Marcoux have investigated the weak amenability of triangular Banach algebras, and Y. Zhang has studied the weak amenability of module extension Banach algebras [23]. Motivated by these earlier investigations, in this section, we study the weak amenability of amalgamated Banach algebra $A \bowtie^{\theta} I$. 
Theorem 6.1. If $A \bowtie^{\theta} I$ is commutative, then $A \bowtie^{\theta} I$ is weakly amenable if and only if $A$ and $I$ are weakly amenable.

Proof. This is immediate by Proposition 2.1, 4, Propositions 2.8.64 and 2.8.65(ii) and Theorem 2.8.69(i)] and noting that $\frac{A \bowtie^{\theta} I}{I} \cong A$.

Example 6.1. Let $G$ be a locally compact abelian group and consider $M(G)=l^{1}(G) \ltimes M_{c}(G)$, the semidirect product of $l^{1}(G)$ and $M_{c}(G)$; see Example2.1(iv). Since $l^{1}(G)$ is weakly amenable, by above theorem, $M(G)$ is weakly amenable if and only if $M_{c}(G)$ is weakly amenable.

In general case, we have one direction of Theorem 6.1 .

Proposition 6.2. If $A$ and $I$ are weakly amenable, then $A \bowtie^{\theta} I$ is also weakly amenable.

Proof. It follows immediately from Proposition 2.1 and [4, Proposition 2.8.65(ii)].

The converse of above proposition does not hold in general. Indeed, it is shown in [15] that the augmentation ideal $I$ of $L^{1}(S L(2, \mathbb{R}))$ is not weakly amenable and that its unitization $I^{\#}$ is weakly amenable.

Example 6.2. Let $G$ be a locally compact group. Since $M(G)=l^{1}(G) \ltimes M_{c}(G)$ and $l^{1}(G)$ is always weakly amenable, by Proposition $6.2, M(G)$ is weakly amenable, provided that $M_{c}(G)$ is weakly amenable.

Example 6.3. Let $G$ be a locally compact group. Then $l^{1}(G) \ltimes L^{1}(G)$ is weakly amenable.

In order to prove a partial converse of Proposition 6.2 we first look at derivations from $A$ to $A^{*}$.

Proposition 6.3. $\mathcal{H}^{1}\left(A, A^{*}\right)$ embeds in $\mathcal{H}^{1}\left(A \bowtie^{\theta} I,\left(A \bowtie^{\theta} I\right)^{*}\right)$.

Proof. Every $D \in \mathcal{Z}^{1}\left(A, A^{*}\right)$ defines a derivation $\tilde{D}: A \bowtie^{\theta} I \rightarrow\left(A \bowtie^{\theta} I\right)^{*}$ by $\tilde{D}(a, i)=$ $(D(a), 0)$, and it can be easily checked that $\tilde{D}$ is inner if and only if $D$ is inner. It follows that the mapping $D \mapsto \tilde{D}$ induces an embedding from $\mathcal{H}^{1}\left(A, A^{*}\right)$ into $\mathcal{H}^{1}\left(A \bowtie^{\theta} I,\left(A \bowtie^{\theta} I\right)^{*}\right)$.

Corollary 6.4. If $A \bowtie^{\theta} I$ is weakly amenable, then so is $A$.

The following corollary has been obtained in [13] with a different method. In fact, we have given a short proof for this result.

Corollary 6.5. ([13, Theorem 2.2]) Let $A$ be a dual Banach algebra. If $A^{* *}$ is weakly amenable, then so is $A$.

Weak amenability of module extension Banach algebras is extensively studied in [23]. We are going to characterize the weak amenability of Banach algebras $A \bowtie^{\text {id }} A$ and $A \oplus_{\phi} B$. 
6.1. Weak Amenability of $A \bowtie^{\text {id }} A$. Here, we focus on the special case $A \bowtie^{\text {id }} A$.

Proposition 6.6. Let $A^{2}$ be dense in $A$. Then $D \in \mathcal{Z}^{1}\left(A \bowtie^{\mathrm{id}} A,\left(A \bowtie^{\mathrm{id}} A\right)^{*}\right)$ if and only if

$$
D(a, b)=\left(D_{1}(a)+D_{2}(b), D_{2}(a)+D_{2}(b)\right) \quad(a, b \in A),
$$

for some $D_{1}, D_{2} \in \mathcal{Z}^{1}\left(A, A^{*}\right)$. Moreover, $D=\operatorname{ad}_{(f, g)}$ if and only if $D_{1}=\operatorname{ad}_{f}$ and $D_{2}=\operatorname{ad}_{g}$, where $f, g \in A^{*}$.

Proof. Let $D: A \bowtie^{\text {id }} A \rightarrow\left(A \bowtie^{\text {id }} A\right)^{*}$ be a derivation. Then we may write

$$
D(a, b)=\left(D_{1}(a)+D_{2}(b), D_{3}(a)+D_{4}(b)\right) \quad(a, b \in A),
$$

where $D_{k}: A \rightarrow A^{*}(1 \leq k \leq 4)$ is a linear operator. If we use the derivation property of $D$ together with the equations (3.1) and (3.3), we get

$$
\begin{aligned}
& \left(D_{1}\left(a_{1} a_{2}\right)+D_{2}\left(a_{1} b_{2}+b_{1} a_{2}+b_{1} b_{2}\right), D_{3}\left(a_{1} a_{2}\right)+D_{4}\left(a_{1} b_{2}+b_{1} a_{2}+b_{1} b_{2}\right)\right)= \\
& \left(a_{1} D_{1}\left(a_{2}\right)+a_{1} D_{2}\left(b_{2}\right)+b_{1} D_{3}\left(a_{2}\right)+b_{1} D_{4}\left(b_{2}\right)+D_{1}\left(a_{1}\right) a_{2}+D_{2}\left(b_{1}\right) a_{2}+D_{3}\left(a_{1}\right) b_{2}+D_{4}\left(b_{1}\right) b_{2},\right. \\
& \left(a_{1}\left[D_{3}\left(a_{2}\right)+D_{4}\left(b_{2}\right)\right]+b_{1}\left[D_{3}\left(a_{2}\right)+D_{4}\left(b_{2}\right)\right]+\left[D_{3}\left(a_{1}\right)+D_{4}\left(b_{1}\right)\right] a_{2}+\left[D_{3}\left(a_{1}\right)+D_{4}\left(b_{1}\right)\right] b_{2}\right) .
\end{aligned}
$$

By setting $a_{1}=a_{2}=0$, we see that $D_{1}, D_{3} \in \mathcal{Z}^{1}\left(A, A^{*}\right)$. By setting $b_{1}=b_{2}=0$ and noting that $A^{2}$ is dense in $A$, we get $D_{2}=D_{4} \in \mathcal{Z}^{1}\left(A, A^{*}\right)$. Also, by choosing $a_{2}=b_{1}=0$, we obtain

$$
D_{2}\left(a_{1} b_{2}\right)=a_{1} \cdot D_{2}\left(b_{2}\right)+D_{3}\left(a_{1}\right) \cdot b_{2},
$$

which implies $D_{2}\left(a_{1}\right) \cdot b_{2}=D_{3}\left(a_{1}\right) \cdot b_{2}$ for all $a_{1}, b_{2} \in A$. Since $A^{2}$ is dense in $A$, it follows that $D_{2}=D_{3}$. The claim about inner derivations can be easily verified.

Theorem 6.7. Let $A^{2}$ be dense in $A$. Then, as vector spaces, we have

$$
\mathcal{H}^{1}\left(A \bowtie^{\text {id }} A,\left(A \bowtie^{\text {id }} A\right)^{*}\right) \cong \mathcal{H}^{1}\left(A, A^{*}\right) \oplus \mathcal{H}^{1}\left(A, A^{*}\right) .
$$

Proof. Define $\varphi: \mathcal{Z}^{1}\left(A, A^{*}\right) \oplus \mathcal{Z}^{1}\left(A, A^{*}\right) \rightarrow \mathcal{Z}^{1}\left(A \bowtie^{\text {id }} A,\left(A \bowtie^{\text {id }} A\right)^{*}\right)$ by $\varphi\left(D_{1}, D_{2}\right)=D$, where

$$
D(a, b)=\left(D_{1}(a)+D_{2}(b), D_{2}(a)+D_{2}(b)\right) \quad(a, b \in A) .
$$

The Proposition 6.6 shows that $\varphi$ is well defined and onto. Since $D$ is inner if and only if $D_{1}$ and $D_{2}$ are inner, according to the Proposition 6.6, $\varphi$ induces the desired isomorphism.

Since every weakly amenable Banach algebra is square dense, we have the following corollary.

Corollary 6.8. The Banach algebra $A \bowtie^{\text {id }} A$ is weakly amenable if and only if $A$ is weakly amenable.

Example 6.4. Let $A$ be a $C^{*}$-algebra or a group algebra of a locally compact group. Then $A \bowtie^{\text {id }} A$ is weakly amenable. 
6.2. Weak Amenability of $A \oplus_{\phi} B$. Let $(a, b) \in A \oplus_{\phi} B$ and $(f, g) \in\left(A \oplus_{\phi} B\right)^{*}$. Then $(a, b) \cdot(f, g),(f, g) \cdot(a, b) \in\left(A \oplus_{\phi} B\right)^{*}$ are given by

$$
\begin{aligned}
& (a, b) \cdot(f, g)=(a \cdot f+g(b) \phi, \phi(a) g+b \cdot g), \\
& (f, g) \cdot(a, b)=(f \cdot a+g(b) \phi, \phi(a) g+g \cdot b) .
\end{aligned}
$$

Proposition 6.9. Let $B^{2}$ be dense in $B$. Then $D \in \mathcal{Z}^{1}\left(A \oplus_{\phi} B,\left(A \oplus_{\phi} B\right)^{*}\right)$ if and only if

$$
D(a, b)=\left(D_{1}(a)+D_{2}(b), D_{4}(b)\right) \quad(a \in A, b \in B),
$$

such that

(i) $D_{1} \in \mathcal{Z}^{1}\left(A, A^{*}\right)$,

(ii) $D_{4} \in \mathcal{Z}^{1}\left(B, B^{*}\right)$,

(iii) $D_{2}: B \rightarrow A$ is a bounded linear map satisfying

(1) $a \cdot D_{2}(b)=D_{2}(b) \cdot a=\phi(a) D_{2}(b)$ for all $a \in A$ and $b \in B$,

(2) $D_{2}\left(b b^{\prime}\right)=\left\langle b, D_{4}\left(b^{\prime}\right)\right\rangle \phi+\left\langle b^{\prime}, D_{4}(b)\right\rangle \phi$ for all $b, b^{\prime} \in B$.

Moreover, $D=\operatorname{ad}_{(f, g)}$ if and only if $D_{1}=\operatorname{ad}_{f}, D_{2}=0$ and $D_{4}=\operatorname{ad}_{g}\left(f \in A^{*}, g \in B^{*}\right)$.

Proof. Let $D: A \oplus_{\phi} B \rightarrow\left(A \oplus_{\phi} B\right)^{*} \cong A^{*} \oplus_{\infty} B^{*}$ be a derivation. Then $D$ is of the form

$$
D(a, b)=\left(D_{1}(a)+D_{2}(b), D_{3}(a)+D_{4}(b)\right) \quad(a \in A, b \in B),
$$

where $D_{1}: A \rightarrow A^{*}, D_{2}: B \rightarrow A^{*}, D_{3}: A \rightarrow B^{*}$ and $D_{4}: B \rightarrow B^{*}$ are linear operators. If we use the derivation property of $D$ together with the equations (6.1) and (6.2), we get

$$
\begin{aligned}
& \left(D_{1}\left(a a^{\prime}\right)+\phi(a) D_{2}\left(b^{\prime}\right)+\phi\left(a^{\prime}\right) D_{2}(b)+D_{2}\left(b b^{\prime}\right), D_{3}\left(a a^{\prime}\right)+\phi(a) D_{4}\left(b^{\prime}\right)+\phi\left(a^{\prime}\right) D_{4}(b)+D_{4}\left(b b^{\prime}\right)\right)= \\
& \left(a D_{1}\left(a^{\prime}\right)+a D_{2}\left(b^{\prime}\right)+\left\langle b, D_{3}\left(a^{\prime}\right)\right\rangle \phi+\left\langle b, D_{4}\left(b^{\prime}\right)\right\rangle \phi, \phi(a) D_{3}\left(a^{\prime}\right)+\phi(a) D_{4}\left(b^{\prime}\right)+b D_{3}\left(a^{\prime}\right)+b D_{4}\left(b^{\prime}\right)\right)+ \\
& \left.\left(D_{1}(a) a^{\prime}+D_{2}(b) a^{\prime}+\left\langle b^{\prime}, D_{3}(a)\right\rangle \phi+\left\langle b^{\prime}, D_{4}(b)\right\rangle \phi, \phi\left(a^{\prime}\right) D_{3}(a)+\phi\left(a^{\prime}\right) D_{4}(b)\right]+D_{3}(a) b^{\prime}+D_{4}(b) b^{\prime}\right) .
\end{aligned}
$$

By setting $b=b^{\prime}=0$ we see that $D_{1} \in \mathcal{Z}^{1}\left(A, A^{*}\right)$ and $D_{3} \in \mathcal{Z}_{\phi}^{1}\left(A, A^{*}\right)$. Letting $a=a^{\prime}=0$ one obtains $D_{4} \in \mathcal{Z}^{1}\left(B, B^{*}\right)$ and $D_{2}\left(b b^{\prime}\right)=\left\langle b, D_{4}\left(b^{\prime}\right)\right\rangle \phi+\left\langle b^{\prime}, D_{4}(b)\right\rangle \phi$.

Now put $a=b^{\prime}=0$. Then we get $b \cdot D_{3}\left(a^{\prime}\right)=0$ in $B^{*}$ which implies $D_{3}=0$ by density of $B^{2}$ in $B$. Hence $D_{2}(b) \cdot a^{\prime}=\phi\left(a^{\prime}\right) D_{2}(b)$. Similarly, choosing $a^{\prime}=b=0$ gives $a \cdot D_{2}\left(b^{\prime}\right)=\phi(a) D_{2}\left(b^{\prime}\right)$.

Using (6.1) and (6.2) one can easily see that $D=\operatorname{ad}_{(f, g)}$ if and only if $D_{1}=\operatorname{ad}_{f}, D_{2}=0$ and $D_{4}=\operatorname{ad}_{g}$.

Let $B$ be a Banach algebra. A derivation $D: B \rightarrow B^{*}$ is called cyclic if

$$
\left\langle b, D\left(b^{\prime}\right)\right\rangle+\left\langle b^{\prime}, D(b)\right\rangle=0 \quad \text { for all } b, b^{\prime} \in B .
$$

We denote by $\mathcal{Z}_{c}^{1}\left(B, B^{*}\right)$ the space of all cyclic derivations which includes $\mathcal{B}^{1}\left(B, B^{*}\right)$. The first cyclic cohomology group of $B$ is $\mathcal{H}_{c}^{1}\left(B, B^{*}\right)=\mathcal{Z}_{c}^{1}\left(B, B^{*}\right) / \mathcal{B}^{1}\left(B, B^{*}\right)$.

Theorem 6.10. $\mathcal{H}^{1}\left(A, A^{*}\right) \oplus \mathcal{H}_{c}^{1}\left(B, B^{*}\right)$ embeds in $\mathcal{H}^{1}\left(A \oplus_{\phi} B,\left(A \oplus_{\phi} B\right)^{*}\right)$. 
Proof. Define $\psi: \mathcal{Z}^{1}\left(A, A^{*}\right) \oplus \mathcal{Z}_{c}^{1}\left(B, B^{*}\right) \longrightarrow \mathcal{Z}^{1}\left(A \oplus_{\phi} B,\left(A \oplus_{\phi} B\right)^{*}\right)$ by $\psi\left(D_{1}, D_{2}\right)=D$, where

$$
D(a, b)=\left(D_{1}(a), D_{4}(b)\right) \quad(a \in A, b \in B) .
$$

It follows from Proposition 6.9 that $D$ is a derivation and it is inner if and only if $D_{1}$ and $D_{2}$ are inner. So $\psi$ induces an injective linear map from $\mathcal{H}^{1}\left(A, A^{*}\right) \oplus \mathcal{H}_{c}^{1}\left(B, B^{*}\right)$ into $\mathcal{H}^{1}\left(A \oplus_{\phi} B,\left(A \oplus_{\phi}\right.\right.$ $\left.B)^{*}\right)$.

Corollary 5.6 of [15] shows that in general $\mathcal{H}^{1}\left(B, B^{*}\right)$ does not embeds into $\mathcal{H}^{1}\left(A \oplus_{\phi} B,\left(A \oplus_{\phi}\right.\right.$ $B)^{*}$ ), and thus it seems that Theorem 6.10 be the best that one could expect.

Corollary 6.11. ([21, Theorem 2.11]) If $A \oplus_{\phi} B$ is weakly amenable, then $A$ is weakly amenable and $B$ is cyclicly amenable.

\section{REFERENCES}

[1] F. Abtahi, A. Ghafarpanah and A. Rejali, Biprojectivity and biflatness of Lau product of Banach algebras defined by a Banach algebra morphism, Bull. Aust. Math. Soc. 91 (2015), 134-144.

[2] R. Arens, The adjoint of a bilinear operator, Proc. Amer. Math. Soc. 2 (1951), 839-848.

[3] S. J. BнAтt and P. A. DABHI, Arens regularity and amenability of Lau product of Banach algebras defined by a Banach algebra morphism, Bull. Aust. Math. Soc. 87 (2013), 195-206.

[4] H. G. DALES, Banach algebras and automatic continuity, Clarendon Press, Oxford, 2000.

[5] H. G. Dales, F. Ghahramani and N. Grønbæk, Derivations into iterated duals of Banach algebras, Studia Math. 128 (1998), 19-54.

[6] H. G. Dales and A. T.-M. LaU, The second duals of Beurling Banach algebras, Mem. Amer. Math. Soc. 177 (836) (2005).

[7] M. D'Anna, C. A. Finocchiaro and M. Fontana, Amalgamated algebras along an ideal, in: Commutative Algebra and Applications, Proceedings of the Fifth International Fez Conference on Commutative Algebra and Applications, Fez, Morocco (2008), W. de Gruyter Publisher, Berlin (2009), 155172.

[8] M. D'Anna, C. A. Finocchiaro and M. Fontana, Properties of chains of prime ideals in an amalgamated algebra along an ideal, J. Pure Appl. Algebra, 214 (2010), 1633-1641.

[9] M. D'Anna, C. A. Finocchiaro and M. Fontana, New algebraic properties of an amalgamated algebra along an ideal, to appear in Comm. Algebra.

[10] M. D'Anna and M. Fontana, An amalgamated duplication of a ring along an ideal: the basic properties, J. Algebra Appl. 6 (2007), 443-459.

[11] M. Eshaghi Gordji and M. Filali, Arens regularity of module actions, Studia Math. 181 (2007), 237-254.

[12] B. E. Forrest and L. W. Marcoux, Weak amenability of triangular Banach algebras, Trans. Amer. Math. Soc. 354 (2002), 1435-1452.

[13] F. Ghahramani and J. LaAli, Amenability and topological centres of the second duals of Banach algebras, Bull. Aust. Math. Soc. 65 (2002), 191-197.

[14] H. Javanshiri and M. Nemati, On a Certain product of Banach algebras and some of its properties, Proc. Rom. Acad. Ser. A, 15 (2014), 219-227.

[15] B. E. Johnson and M. C. White, A non-weakly amenable augmentation ideal, Preprint.

[16] E. Kaniuth, A Course in Commutative Banach Algebras, Springer, New York, 2009.

[17] A. T.-M. LAU, Analysis on a class of Banach algebras with applications to harmonic analysis on locally compact groups and semigroups, Fund. Math. 118 (1983), 161-175. 
[18] A. T.-M. LAU and V. Losert, On the second conjugate algebra of $L^{1}(G)$ of a locally compact group, J. London Math. Soc. 37 (1988) 464-470.

[19] V. Runde, Lectures on amenability, Lecture Notes in Mathematics, Springer, Berlin, 2002.

[20] P. Sahandi, N. Shirmohammadi and S. Sohrabi, Cohen-Macaulay and Gorenstein properties under the amalgamated construction, Comm. Algebra, 44 (3) (2016), 1096-1109.

[21] M. SAngani Monfared, On certain products of Banach algebras with applications to harmonic analysis, Studia Math. 178 (3) (2007), 277-294.

[22] M. P. Thomas, Principal ideals and semi-direct products in commutative Banach algebras, J. Funct. Analysis 101 (1991), 312-328.

[23] Y. Zhang, Weak Amenability of Module Extensions of Banach Algebras, Trans. Amer. Math. Soc. 354 (2002), 4131-4151.

Department of Mathematics, University of Tabriz, Tabriz, Iran.

E-mail address: h_p_aghababa@tabrizu.ac.ir, h_pourmahmood@yahoo.com

E-mail address: shirmohammadi@tabrizu.ac.ir 